\title{
State of the Journal 2010: Managing Growth, Extending Our Reach-Now the Official Journal for the Society of Surgical Oncology and the American Society of Breast Surgeons
}

\author{
Mark Roh, MD ${ }^{1}$, Deborah Whippen ${ }^{2}$, and Charles Balch, $\mathbf{M D}^{3}$ \\ ${ }^{1}$ Department of Surgery, MD Anderson Cancer Center Orlando, Orlando, FL; ${ }^{2}$ Annals of Surgical Oncology, Editorial \\ Office, Orange Park, FL; ${ }^{3}$ Department of Surgery, John Hopkins Medical Center, Baltimore, MD
}

We begin this first issue of 2010 by welcoming the 3,000 members of the American Society of Breast Surgeons and assessing the growth of the Annals of Surgical Oncology.

The Annals is now the official journal for both the Society of Surgical Oncology and the American Society of Breast Surgeons (ASBS). The ASBS brings with it a membership whose mission aligns nicely with the strategic vision of the Society of Surgical Oncology (SSO) and the Annals. The ASBS will have a special ASO edition to be published in October 2010, which will be a valuable additional resource for both ASBS members and SSO members.

The growth of the Annals, both in size and content, has been impressive! Over the last 5 years, submissions and resubmissions of original work have almost tripled (approximately 600 articles submitted in 2005-2006 vs. more than 1600 in 2008-2009). The number of reviewers volunteering their critical expertise to the peer review process grew from 750 in 2006 to over 2000 experts in 2009 , with an average number of reviews completed per reviewer declining from 2.5 to 1.6. This growth in the number of peer review experts and the range in their expertise reflects the journal's continuing expansion of breadth, depth, and range of subject matter. We thank them for their voluntary effort that shapes the scientific excellence of the journal.

The Annals now covers 18 content areas, as reflected in the structure of the Editorial Board (Table 1). New

(C) Society of Surgical Oncology 2010

Published Online: 16 December 2009

Annals of Surgical Oncology, Editorial Office

e-mail: info@asoeditorial.org additions in 2009 include a section on Colorectal Cancer led by Heidi Nelson, MD, and an expansion of Translational Research to include Biomarkers, led by James Cusack, MD. The journal continues to be especially strong in Hepatobiliary and Pancreatic Tumor papers, now broken into two distinct groups led by Michael Choti, MD, and Andrew Lowy, MD, respectively. Gastrointestinal, Melanoma, and Breast Oncology sections continue to grow as well.

\section{STRATEGIC RELATIONSHIPS}

This successful relationship with ASBS and the Annals provides a model for the establishment of other educational partnerships with societies worldwide. Relationships have also been established with Latin American Federation of Surgery, the Hellenic Society of Surgical Oncology, and the Society of Oncology and Cancer Research in Nigeria. The goal of pursuing these partnerships is to increase visibility, usage, and citations of the journal. A number of other international societies have expressed interest in developing such a relationship with the journal, and discussions are continuing with the editors and the SSO leadership.

\section{INTERNATIONAL REACH}

Manuscript submissions from North America continue to be the primary source of content. However, the Annals has experienced worldwide growth in manuscript submissions, with a particular increase from Asia and Europe. Likewise, online access by readers has grown substantially beyond the borders of North America. Downloads of manuscripts from the Web site indicate that nearly two- 
TABLE 1 2009-2010 Editorial Board sections and section editors

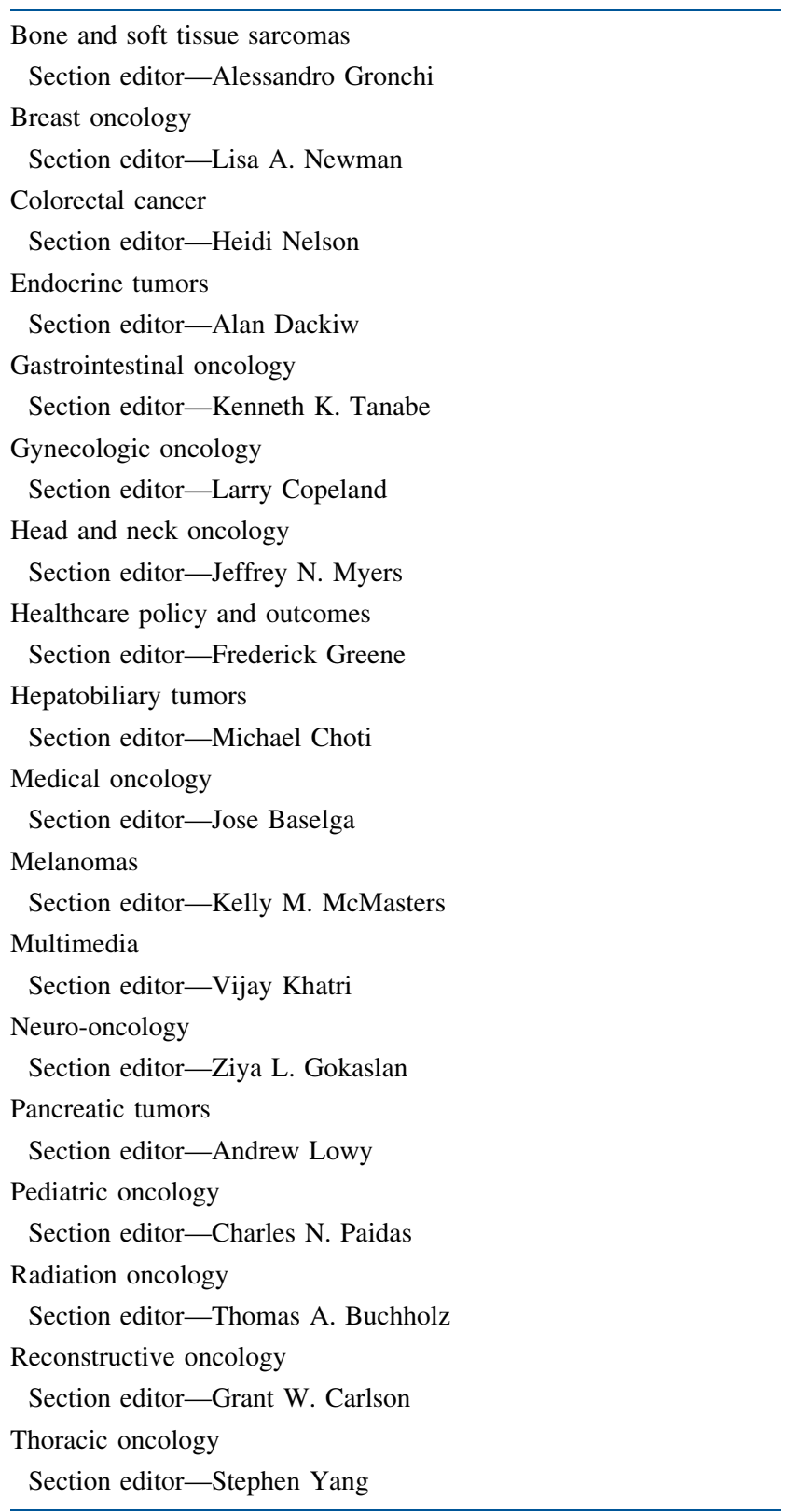

thirds of our readers are from countries other than the United States, with primary concentrations in Europe and Asia. International participation in the journal reflects the significance of Annals in the research arena globally; the journal is pleased to see its position as an educational resource extended to surgeons and other readers around the globe.

\section{VISION, MISSION, AND EDITORIAL LEADERSHIP}

Vision and mission statements for the Annals were drafted by editors and approved by the SSO Executive Council in fall 2008 (Table 2). These statements of identity
TABLE 2 Strategic plan: vision and mission

Vision

The Annals of Surgical Oncology (ASO) promotes high-quality surgical oncology management by communicating advances in research and education that are relevant and valuable to the provision of contemporary multidisciplinary care for patients with cancer. ASO, the official journal of the Society of Surgical Oncology (SSO), is the journal of choice of surgical oncologists worldwide regarding surgical oncology research and education

Mission

The mission of the journal is to serve its readers by:

1. Representing and advancing the profession of surgical oncology throughout the nation and the world

2. Promoting the highest quality multidisciplinary patient care and practice management

3. Providing relevant cancer education and research training materials using print and electronic media

4. Promoting clinical and translational cancer research, with an emphasis on clinical trials

5. Facilitating the career development of surgical trainees and their transition into academic and community-based practice

6. Promoting public policy and patient advocacy issues related to surgical patient with cancer

and purpose represent the journal's commitment to the highest level of quality. Journal editors recently revisited the Annals Strategic Plan to again prioritize the activities relevant to each of the Plan's six goals, which are structured in the following areas: Goal 1, Multispecialty Member Needs; Goal 2, Education and Training; Goal 3, Clinical and Translational Research; Goal 4, Career Development; Goal 5, Quality of Cancer Care and Practice Management; and Goal 6, Governance and Operations. Annals is active in all these goal areas, and the editors continue to track and assess related progress and accomplishments.

\section{RESOURCES}

Efficient use of resources has become an important topic for the journal. In addition to the journal's availability on the publisher's Web program, SpringerLink, Annals articles and other journal information are now also online on the journal's new portal, http://www.annsurgoncol.org/. The portal is designed to be an easy and intuitive search tool for individual readers and to be a delivery vehicle for the most important and up-to-date journal information.

The journal's Editorial Office is staffed under the leadership of Deb Whippen (info@asoeditorial) and two editorial staff-Julie Leneghan and Joe Wiggs-who have accommodated the significant increase in manuscript volume (Fig. 1) and its related author, reviewer, and editor support needs. The publishing program for Annals has also grown through the journal's association with Springer and 


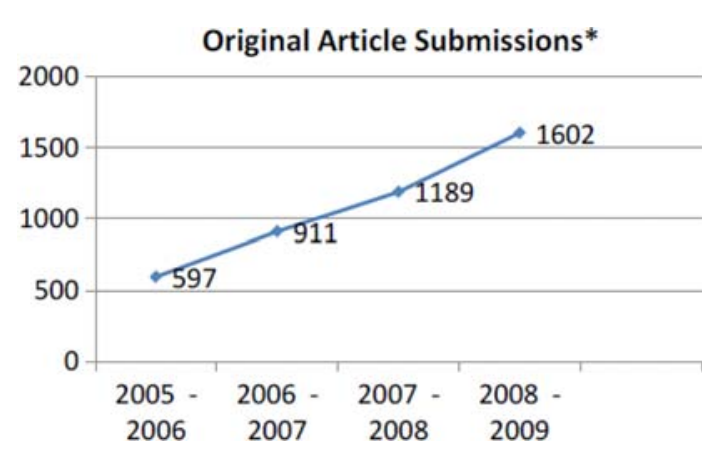

FIG. 1 Three-year comparison of original article submissions to Annals, including revisions. There were 1,880 total submissions from July 1, 2008 to June 30, 2009, including editorials, streaming videos, and letters-to-the-editor

the able leadership and expertise of Annie Cimino, Yvonne Chan, Anna Uger, and Ben Ellis.

Regarding the print issues, the Annals published fewer pages in 2009 than in either of the previous 2 years. The tripling of manuscript submissions since 2006 has improved the quality, but it has become a challenge to manage the page budget. The Annals will fulfill its mission through publication of the highest-quality articles, and the continuing growth of submissions will mean that more papers will be not found acceptable for publication.

A new policy to be effective in 2010 is to enforce a word limit for manuscripts and to limit the number of tables and figures per paper. As the Instruction for Authors in this issue shows, the word limit for journal articles is now 3000 words with a maximum of six tables and figures. We thank authors in advance for their adherence to these new guidelines and encourage them to send us their very best research. We also thank reviewers for helping the editors of the journal assess the priority of each manuscript as well as its quality and the economy with which manuscripts deliver their message.

The continuing partnership of the journal with SSO and ASBS members, the publisher Springer, expert authors, critical reviewers, and devoted members of the Editorial Board, including section editors and associate editors, defines its scientific and educational impact, that in turn influences multidisiciplinary cancer care worldwide, both in 2010 and in the years to come. We thank the many people who continue to contribute to the Annals' success. 